\title{
Erratum \\ Specific Inhibition of Phosphodiesterase-4B Results in Anxiolysis and Facilitates Memory Acquisition
}

Alexander McGirr, Tatiana V Lipina, Ho-Suk Mun, John Georgiou, Ahmed H Al-Amri, Enoch Ng, Dongxu Zhai, Christina Elliott, Ryan T Cameron, Jonathan GL Mullins, Fang Liu, George S Baillie, Steven J Clapcote and John C Roder

Neuropsychopharmacology (2017) 42, II 78; doi:1 0.1038/npp.2016.263

Correction to: Neuropsychopharmacology (2016) 41, 1080-1092; doi:10.1038/npp.2015.240; published online 2 September 2015
This article was originally published under NPG's License to Publish, but has now been made available under a [CC BY 4.0] license. The PDF and HTML versions of the paper have been modified accordingly. 